\title{
What are the Appropriate Surgery and Postoperative Surveillance for Intraductal Papillary Mucinous Neoplasm?
}

\author{
Noboru Ideno, Kohei Nakata, Masafumi Nakamura \\ Department of Surgery and Oncology, Graduate School of Medical Sciences, Kyushu University, Fukuoka, Japan
}

Received May 16, 2021

Revised May 24, 2021

Accepted May 24, 2021

Corresponding author:

Masafumi Nakamura

E-mail: nakamura.masafumi.861@

m.kyushu-u.ac.jp

https://orcid.org/0000-0002-6196-8643
Although many guidelines for pancreatic cystic neoplasms focus on the management of intraductal papillary mucinous neoplasm of the pancreas (IPMN) at the highest oncological risk, there are many issues that surgeons need to consider at the time to plan the surgical procedures based on characteristics of IPMN subtypes, such as multiplicity of branch duct-IPMN (BDIPMN) and intraductal spreading of main duct-IPMN (MD-IPMN). For multifocal BD-IPMN, partial pancreatectomy would be selected to remove BD-IPMN with predictors of malignancy, while the other lesions without risk factors can be left, although total pancreatectomy might be considered if the patients have a strong family history of pancreatic cancer. Partial pancreatectomy would be also adequate procedure for MD-IPMN if negative surgical margin for highgrade dysplasia or invasive carcinoma were achieved. It has become to be well-known that patients with BD-IPMN are at increased risk for developing not only IPMN-associated pancreatic ductal adenocarcinoma (PDAC) but also PDAC independent from the IPMN. Hence, the detection of a concomitant PDAC is also an important focus for strategies after resection of BDIPMNs. Our recent analysis of patients after partial pancreatectomy for MD-IPMN with negative surgical margin identified an unexpected recurrence pattern, which we called "monoclonal skip" recurrence. MD-IPMN seems to be disseminated in the pancreatic ductal systems and MD-IPMN with identical genetic background was detected in the remnant pancreas even in a long time after index surgery. We proposed strategies of postoperative surveillance based on characteristics and natural history of each morphological subtype.

Key Words: Intraductal papillary mucinous neoplasms of the pancreas; Guideline; Recurrence

\section{INTRODUCTION}

Intraductal papillary mucinous neoplasm of the pancreas (IPMN) is a bona fide precursor lesion of pancreatic ductal adenocarcinoma. Similar to the well-defined adenoma-carcinoma sequence in colorectal cancer and pancreatic ductal adenocarcinoma, IPMNs seem to follow a pattern progressing from IPMN low-grade (LG), to IPMN high-grade (HG), and eventually IPMN with an associated invasive carcinoma (invasive IPMN). According to a pathological examination of resected IPMNs, the distinction of involved pancreatic duct seems to be important because main duct-IPMN (MDIPMN), whether symptomatic or not, have a high rate of malignancy, whereas branch duct-IPMN (BD-IPMN), which often are incidentally discovered, have a much lower rate.

These findings led to the publication of guidelines, like the Sendai guidelines, recommending resection for all $\mathrm{MD}$ IPMN, but observation for asymptomatic BD-IPMNs that have a low risk of malignancy. Although strategies for IPMN at the highest risk of malignancy has been clearly described in guidelines, several issues need to be discussed, such as determination of transection lines in surgery for MD-IPMN and management of BD-IPMN left in the remnant pancreas. In addition, as an increased number of patients with IPMN were appropriately managed based on the guidelines, 
the increased number of patients who were longitudinally surveyed with or without resection led to the publication of many studies demonstrating the development of high-risk lesions in the pancreas with IPMN with lower risk or remnant pancreas of patients who underwent partial pancreatectomy.

In this review, we provide an overview of current insights into studies focusing on the characteristic of IPMN which surgeons need to know for decision making in the management of multifocal BD-IPMNs and diffuse type MD-IPMN, and methods of post-operative surveillance considering patterns of recurrence according to the types of IPMN.

\section{DEFINITION OF TERMINOLOGIES}

\section{Macroscopic Morphological Classification}

IPMNs are divided into three morphological types including main duct type (MD-IPMN), branch duct type (BDIPMN), and mixed type (Fig. 1). The classification should be determined by radiological findings obtained by imaging studies, most clearly by magnetic resonance cholangiopancreatography (MRCP). This review refers to the classification defined in the International Association of Pancreatology 2017 (IAP 2017) [1]. MD-IPMN is characterized by segmental or diffuse dilation of the main pancreatic duct of $>5 \mathrm{~mm}$ without other causes of obstruction. Pancreatic cysts of $>5 \mathrm{~mm}$ in diameter that communicate with the main pancreatic duct (MPD) should be considered as BDIPMN. Mixed-type IPMN meets the criteria for both MD-
IPMN and BD-IPMN.

\section{Histopathologic Grade of IPMN and Invasive Carcinoma Arising in the Pancreas with IPMNs}

In this review, the histopathologic grade will be stated as proposed in the Baltimore consensus meeting for neoplastic precursor lesions in the pancreas [2]. Precursor lesions to invasive carcinoma are classified into Pancreatic intraepithelial neoplasia (PanIN)/IPMN LG and HG. PanIN LG and PanIN HG are equal to PanIN-1, 2, and PanIN-3, respectively. While IPMN LG and HG are qual to IPMN with low-grade/intermediate-grade dysplasia and high-grade dysplasia, respectively, which were defined in the 2010 World Health Organization (WHO) classification for tumors of the pancreas. When invasive carcinoma is identified in the pancreas with IPMN, the relationship between invasive carcinoma and IPMN will be carefully examined to clarify the precursor of invasive carcinoma. Invasive carcinoma which arises from an IPMN is "IPMN with an associated invasive carcinoma”, hereinafter called “invasive IPMN" (Fig. 2A, 2B). Invasive carcinoma can also arise independently in a pancreas containing IPMNs. This will be referred to as "invasive carcinoma concomitant with IPMN" (Fig. 2C-2F). As it is still controversial if PanIN/IPMN HG should be called "malignancy", precursor lesions with high-grade dysplasia will be called "high-risk lesions" in this review, as we have previously described [3].
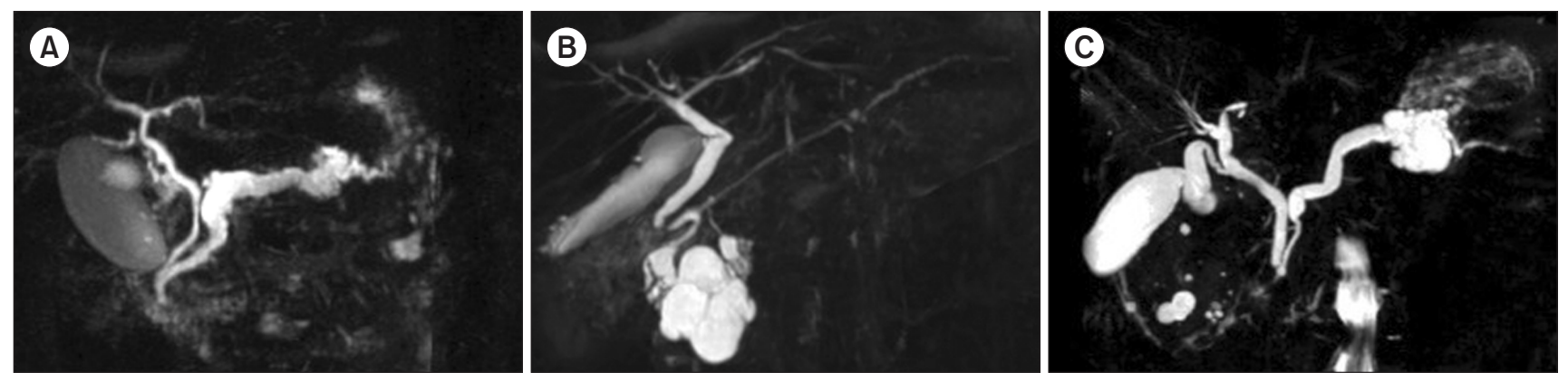

Fig. 1. Three morphological subtypes of intraductal papillary mucinous neoplasm of the pancreas (IPMN) demonstrated by magnetic resonance cholangiopancreatography. Main duct IPMN (A), branch duct-IPMN (B), mixed type-IPMN (C). 

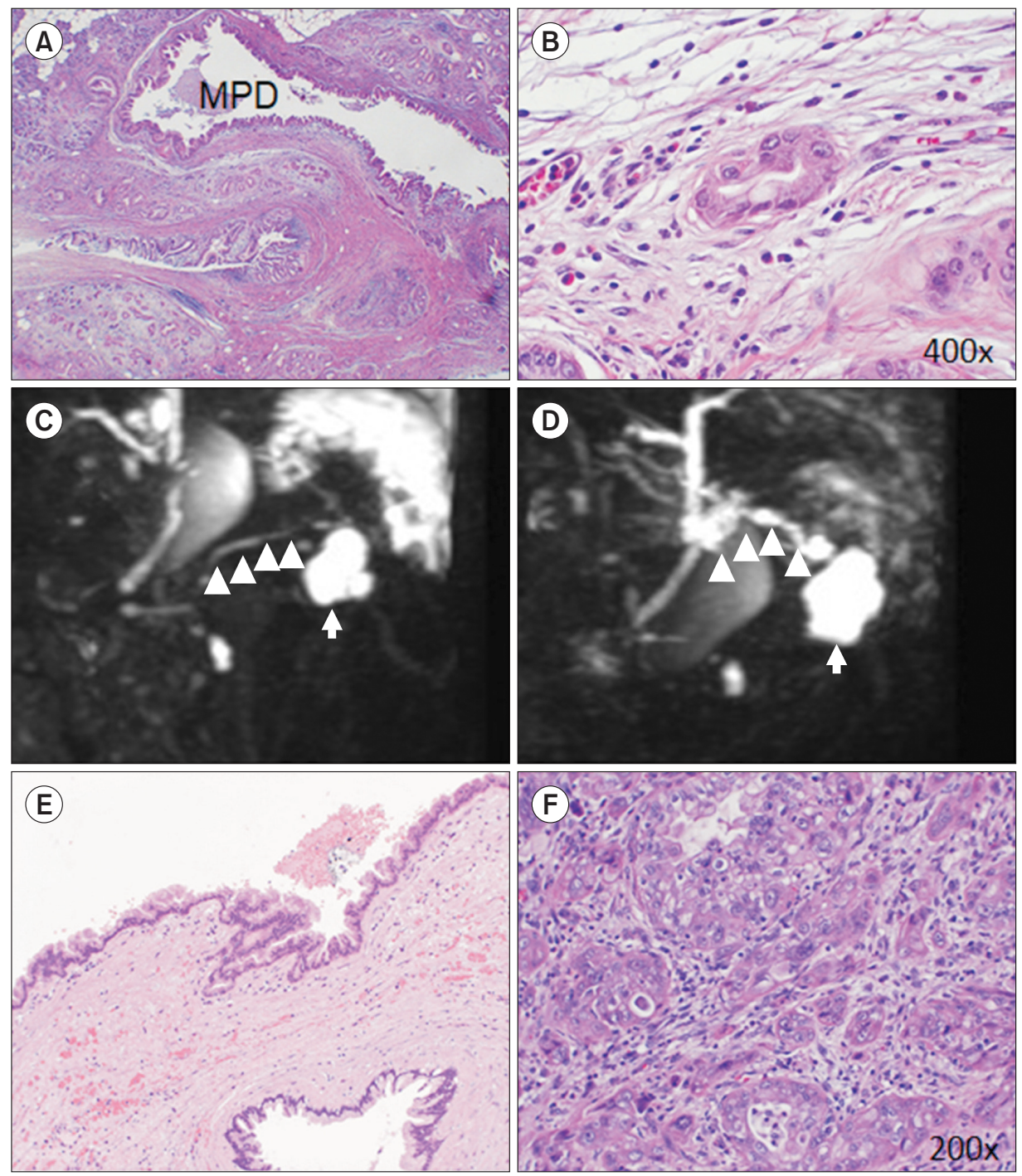

Fig. 2. Distinction of IPMN with an associated invasive carcinoma (invasive IPMN) and invasive carcinoma concomitant with IPMN. MDIPMN with a tubular duct invading into pancreatic parenchyma (A, B). MRCP demonstrating BD-IPMN (white arrow) without MPD dilation (white arrowheads) (C). Development of pancreatic ductal carcinoma in the pancreatic body led to dilation of MPD (white arrowheads) not associated with BD-IPMN (white arrow) (D). Histopathologic assessment confirmed BD-IPMN-low grade (E) and invasive carcinoma concomitant with IPMN (F). IPMN, intraductal papillary mucinous neoplasm; MD-IPMN, main duct IPMN; BD-IPMN, branch ductIPMN; MRCP, magnetic resonance cholangiography; MPD, main pancreatic duct.

\section{SURGICAL MANAGEMENT FOR IPMN}

Since IPMNs display various degrees of dysplasia following adenoma-carcinoma sequencing, dilated main pancreatic duct or pancreatic cyst suspected for IPMN will be initially investigated by physicians to predict the disease progression. Surgery will be recommended for all surgically fit patients who have IPMN with invasive features. Invasive IPMNs are classified into tubular adenocarcinoma and colloid carcinoma. Invasive IPMN with tubular subtypes essentially behave like conventional invasive ductal carcinoma, while those with colloid subtype have favorable prognosis [4,5]. Invasive IPMNs with either subtype should be removed by radical pancreatectomy with lymph node dissection as well as conventional pancreatic ductal adenocarcinoma (PDAC), because 45 and 31\% of invasive IPMN patients had T3/4 diseases and lymph node metastasis, respectively, according to a recent nationwide survey in Japan [6].

Patients with IPMN who underwent surgery before the IPMN became invasive carcinoma displayed significantly longer survival time $[7,8]$, and therefore, management strategy is rather essential for patients who have IPMNs without invasive features to determine optimal timing for surgery.

The management of IPMN is based on morphological and clinical parameters because the risk of malignant transformation is not accurately predictable. Three major guidelines, including IAP 2017 [1], European evidence-based guidelines on pancreatic cystic neoplasms (EURO) [9], and American 
Gastroenterological Association 2015 guidelines (AGA) [10] referred to diagnosis, treatment and surveillance of IPMN, as summarized in Table 1.

The IAP 2017 are most comprehensive and practical for decision making in the management of IPMN [1]. Surgical resection of MD-IPMN is strongly recommended for all surgically fit patients with MPD $>10 \mathrm{~mm}$, jaundice, or mural nodules. Although the lower incidence of malignancy in BDIPMN might lead the more conservative management of the patients with the disease, those with "high-risk stigmata", including obstructive jaundice, enhancing mural nodule $\geq 5$ $\mathrm{mm}$, and main pancreatic duct $\geq 10 \mathrm{~mm}$ will be considered surgery if clinically appropriate. The final decision should be individualized and depends not only on the risk of malignancy but on the patient's life expectancy, comorbidities and extent of MD dilation/cyst location in those with either subtype of IPMNs. Surveillance is necessary for early detection of malignant progression of IPMN and concomitant pancreatic cancer both in patients on observation and after resection of IPMN.

There are some differences in the management of three subtypes of IPMN between IAP 2017 and EURO guidelines. Although IAP 2017 does not specifically describe management of mixed type IPMN, EURO guidelines advise resection of mixed type-IPMN as well as MD-IPMN with the same threshold of main duct size as that in IAP 2017. Indication for resection of IPMN in EURO guidelines also has 2 categories of indication for resection of IPMN including "absolute indications" and "relative indications", which are similar to "high-risk stigmata" and "worrisome features", respectively, in IAP 2017 as presented in Table 1. The AGA suggests that patients with both a solid component and a dilated pancreatic duct and/or concerning features on endoscopic ultrasound guided fine needle aspiration biopsy should undergo surgery to reduce the risk of mortality from carcinoma.

Table 1. Comparison of 3 Guidelines Stating Indications for Surgery and Surveillance of Resected IPMNs

\begin{tabular}{|c|c|c|c|}
\hline Guidelines & $\begin{array}{l}\text { International consensus } \\
\text { guidelines } 2017 \text { (IAP) }\end{array}$ & $\begin{array}{l}\text { European evidence-based } \\
\text { guidelines on pancreatic } \\
\text { cystic neoplasms (EURO) }\end{array}$ & $\begin{array}{c}\text { American Gastroenterological } \\
\text { Association Institution } \\
\text { Guideline (AGA) }\end{array}$ \\
\hline Types of guideline & Consensus guidelines & Evidence-based guidelines & Evidence-based guidelines \\
\hline $\begin{array}{l}\text { Type of tumor/ } \\
\text { neoplasm }\end{array}$ & IPMN and MCN & Cystic tumors of the pancreas & $\begin{array}{l}\text { Asymptomatic neoplastic } \\
\text { pancreatic cyst }\end{array}$ \\
\hline $\begin{array}{l}\text { Indication for } \\
\text { resection }\end{array}$ & $\begin{array}{l}\text { High-risk stigmata: obstructive } \\
\text { jaundice, enhancing mural nodule, } \\
\text { MPD } \geq 10 \mathrm{~mm} \text { ) Worrisome features: } \\
\text { pancreatitis, cyst } \geq 3 \mathrm{~cm} \text {, enhancing } \\
\text { mural nodule }<5 \mathrm{~mm} \text {, thickened/ } \\
\text { enhancing cyst walls, main duct size } \\
5-9 \mathrm{~mm} \text {, abrupt change in caliber of } \\
\text { pancreatic duct, lymphadenopathy, } \\
\text { increase serum level of CA19-9, cyst } \\
\text { growth } \geq 5 \mathrm{~mm} / 2 \text { years } \\
\text { EUS findings: definite nodule, MPD } \\
\text { involvement, positive cytology }\end{array}$ & $\begin{array}{l}\text { Absolute indications: positive cytology } \\
\text { for malignant/high grade dysplasia, } \\
\text { solid mass, jaundice, enhancing mural } \\
\text { nodules ( } \geq 5 \mathrm{~mm}) \text {, main pancreatic } \\
\text { dilation } \geq 10 \mathrm{~mm} \text {. } \\
\text { Relative indications: grow-rate } \geq 5 \mathrm{~mm} / \\
\text { year, increased levels of serum CA19- } \\
9 \text { ( } \geq 37) \text {, main pancreatic duct dilation } \\
\text { from 5-9.9 mm, cyst diameter } \geq 40 \\
\text { mm, new-onset diabetes mellites, } \\
\text { acute pancreatitis (caused by IPMN), } \\
\text { enhancing mural nodules ( }<5 \mathrm{~mm})\end{array}$ & $\begin{array}{l}\text { Two or more high-risk features } \\
+ \text { positive cytology in EUS- } \\
\text { FNA }\end{array}$ \\
\hline $\begin{array}{l}\text { Surveillance of } \\
\text { resected cases }\end{array}$ & $\begin{array}{l}\text { Invasive IPMN: same surveillance as } \\
\text { PDAC; noninvasive IPMN (without } \\
\text { residual lesion): repeat examination } \\
\text { at } 2 \text { and } 5 \text { years }\end{array}$ & $\begin{array}{l}\text { Invasive IPMN: same surveillance } \\
\text { as PDAC; IPMN with high-grade } \\
\text { dysplasia or MD-IPMN: follow-up } \\
\text { every } 6 \text { months for the first } 2 \text { years, } \\
\text { followed by yearly surveillance. } \\
\text { IPMN with low-grade dysplasia: same } \\
\text { as non-resected IPMN }\end{array}$ & $\begin{array}{l}\text { Cyst with invasive cancer or } \\
\text { dysplasia: MRI every } 2 \text { years; } \\
\text { cyst without high-grade } \\
\text { dysplasia or malignancy: no } \\
\text { routine surveillance }\end{array}$ \\
\hline
\end{tabular}

IPMN, intraductal papillary mucinous neoplasm; MCN, mucinous cystic neoplasms; MPD, main pancreatic duct; PDAC, pancreatic ductal adenocarcinoma; CA19-9, carbohydrate antigen 19-9; MRI, magnetic resonance imaging; EUS, endoscopic ultrasound; FNA, fine needle aspiration. 


\section{BD-IPMN}

There are several biological characteristics of BD-IPMN that surgeons need to understand to determine types of procedure and surveillance methods. This section contains the frequency of malignancy of BD-IPMN, multiplicity of BDIPMNs, and risk stratification of patients with BD-IPMN based on family history of PDAC.

\section{Malignant Transformation of BD-IPMN}

The frequency of invasive carcinoma and HG-IPMN in resected BD-IPMN varies ranging from 14.4 to $47.9 \%$, and that of invasive cancer is $18.5 \%(6.1-37.7 \%)$ in recent series [1]. Although there is no prospective study that surveyed patients with BD-IPMN for a long time, a large case series including 1,404 patients with BD-IPMN demonstrated that the 5-year incidence rate of IPMN-derived carcinomas and pancreatic adenocarcinomas was 3.3\%, reaching $12 \%$ at 15 years after IPMN diagnosis [11]. These findings indicated that a minor subset of BD-IPMNs has the potential to progress into high-risk lesions and the risk of malignancy increases according to the time course, supporting the recommendation of the lower threshold for surgery in younger IPMN patients in guidelines [1].

\section{Multiplicity of BD-IPMN}

The prevalence of multifocality in IPMNs varies, ranging from 25 to $45 \%$ [11-14]. Since partial pancreatectomy is recommended to remove BD-IPMN with the highest oncologi- cal risk, BD-IPMNs will be left in the remnant pancreas of patients with multifocal BD-IPMNs. There is some evidence including ours which suggests acceptance of leaving BDIPMNs without risk factors. Mori et al. [15] reported that residual lesions after partial resection for multifocal IPMN did not show significant progression and disease specific survival after partial resection was comparable between multifocal BD-IPMN and solitary BD-IPMN. In addition to the clinical data, molecular/pathological analyses also support this conservative management of multifocal BD-IPMN in the remnant pancreas. Matthaei et al. [16] assessed the histological subtypes and the clonal relationships among multifocal BD-IPMNs using KRAS mutational analyses and loss of heterozygosity ( $\mathrm{LOH}$ ) analyses. The majority of multifocal BD-IPMNs exhibited a gastric-subtype with low to intermediate dysplasia, and they identified nonoverlapping KRAS gene mutations and discordant $\mathrm{LOH}$ profiles among multifocal BD-IPMNs in the same pancreas. These findings indicated that the majority of multifocal IPMNs arise independently and possess a low risk of malignancy.

\section{BD-IPMNs with Familial History of Pancreatic Cancer}

There is some evidence suggesting the possible presence of high-risk lesions in the pancreas with benign IPMN in high-risk patients (Table 2). Shi et al. [17] examined resected pancreata from patients with a strong family history of pancreatic cancer. They found that noninvasive precursor lesions at higher grades were common in patients with a strong family history of pancreatic cancer than in those with sporadic disease. Multifocal small benign BD-IPMN might

Table 2. Studies Investigated IPMN Patients with Family History of Pancreatic Cancer and Germline Mutations in Patients with IPMN

\begin{tabular}{|c|c|c|c|c|c|}
\hline Author & Journal & Year & Subject & No. of subject & Main findings \\
\hline $\begin{array}{l}\text { Shi et al. } \\
\text { [17] }\end{array}$ & Clin Cancer Res. & 2009 & $\begin{array}{l}\text { Patients with strong family } \\
\text { history of pancreatic cancer }\end{array}$ & 51 & $\begin{array}{l}\text { PanIN/IPMN were of a higher grade } \\
\text { in patients with strong family history } \\
\text { pf pancreatic cancer }\end{array}$ \\
\hline $\begin{array}{l}\text { Bartsch et al. } \\
\text { [18] }\end{array}$ & Fam Cancer & 2013 & $\begin{array}{l}\text { Patients with family history of } \\
\text { pancreatic cancer or } B R C A 2 / \\
P A L B 2 \text { germline mutations }\end{array}$ & 125 & $\begin{array}{l}\text { Multifocal BD-IPMNs indicate } \\
\text { presence of PanIN high-grade }\end{array}$ \\
\hline $\begin{array}{l}\text { Skaro et al. } \\
{[19]}\end{array}$ & Gastroenterology & 2019 & Patients with IPMNs & 315 & $\begin{array}{l}23 \text { patients had germline mutations } \\
\text { associated with cancer risk. }\end{array}$ \\
\hline
\end{tabular}

IPMN, intraductal papillary mucinous neoplasm; BD-IPMN, branch duct-IPMN. 
be a predictor of the presence of higher grade PanIN in patients with a family history of pancreatic cancer. Bartsch et al. [18] reported 4 cases of high-risk patients with a family history of pancreatic cancer who had benign multifocal BDIPMNs and identified PanIN-HG lesions in the elsewhere of the pancreas of all 4 patients. Skaro et al. [19] sequenced 94 genes with variants associated with cancer risk in DNA obtained from nontumor tissue samples from 315 patients with histologically confirmed IPMN. The reported that $7.3 \%$ of IPMN patients had germline mutations associated with cancer risks, such as ATM, BRCA2, MSH6, and PALB2 gene mutations. Patients with IPMNs and germline mutations associated with pancreatic cancer were more likely to have concurrent invasive carcinoma compared with those with IPMNs without these mutations. Based on these, IAP guideline suggested that the threshold for total pancreatectomy for IPMN should be lowered in patients with a strong family history of PDAC.

\section{Summary of Surgery for BD-IPMN}

For adequate management of multifocal BD-IPMN, partial pancreatectomy is indicated in lesions with the highest risks, while those without risks should be followed up. Prophylactic total pancreatectomy for multifocal BD-IPMN is not always recommended, except for patients with a family history of pancreatic cancer.

\section{MD-IPMN}

Resection of MD-IPMN aims to achieve complete removal of a tumor with a negative margin. Although it might be relatively easy to determine the resection side and transection line in MD-IPMN with segmental dilation of the main pancreatic duct or diffuse type with focal lesions, such as mural nodules or dilated branch lesions. On the other hand, a careful evaluation would be necessary for the diffuse dilation type without focal lesions. In this section, the frequency of malignancy of MD-IPMN and methods to determine the transection line pre-/intra-operatively would be demonstrated.

\section{Malignant Transformation of MD-IPMN}

The mean frequency of invasive carcinoma and highgrade dysplasia in MD-IPMN is $61.6 \%$, range from 36 to $100 \%$, and the mean frequency of invasive IPMN is $43.1 \%$, range from 11 to $81 \%$ [1]. It is clear that MD-IPMN harbors a higher risk of malignancy compared to BD-IPMN. Although several studies have described clinical and radiologic features that are common in MD-IPMN carcinoma, malignancy predictors in MD-IPMN are shared with BD-IPMN in IAP 2017 [1].

\section{Methods to Determine Transection Line in Surgery for MD-IPMN}

Three studies demonstrated that there was no difference in recurrence between margin positive and margin negative mostly for LG [20-22]. Marchegiani et al. [23] reported that a significantly higher recurrence rate in MD-IPMN patients with margin positive for HG/invasive carcinoma. Tamura et al. [24] showed margin positive for LG or recurrence in the remnant pancreas did not worsen prognosis in MD-IPMN patients. Based on these studies, partial pancreatectomy can be planned in poor risk patients with diffuse type MDIPMN, if it was possible to accurately differentiate HG from LG in an intraductal tumor.

Peroral pancreatoscopy (POPS) can detect superficial spread lesions in MPD and targeting biopsy using POPS might be helpful for the preoperative assessment to predict the extent of intraductal spreading of the tumor $[25,26]$. However, unfortunately, our recent case series demonstrated that the sensitivity of targeting biopsy during POPS to diagnose HG dysplasia was $0 \%$ [27].

\section{Summary of Surgery for MD-IPMN}

In summary, partial pancreatectomy for MD-IPMN with negative surgical margin for $\mathrm{HG}$ or invasive carcinoma is adequate. The presence of LG at the cut end seems not to be associated with clinically relevant recurrence. Although POPS might be useful for the determination of the transec- 
tion line for the partial pancreatectomy for MD-IPMN, the significance of intraoperative pathological assessment has not been changed yet.

\section{POSTOPERATIVE SURVEILLANCE}

\section{Postoperative Surveillance in Guideline}

Methods of postoperative surveillance in three guidelines were summarized in Table 1. IAP 2017 and EURO guidelines recommend continuation of surveillance as long as the patient remains fit for surgery irrespective of histopathological grade or margin status of initially resected IPMN, because the patient may benefit from additional pancreatectomy for remnant pancreatic lesions.

\section{Patterns of Recurrence or Development of New Lesions after Pancreatectomy for IPMN}

The recommendations of surveillance after resection of IPMN by guidelines led to many studies reporting a recurrence of IPMN or development of new high-risk lesions in the remnant pancreas. Studies reported remnant pancreatic lesions after resection of IPMN are summarized in Table 3 [3,28-35]. Of note, rates of the patients who developed overall and malignant remnant pancreatic lesion were 5 to $22 \%$ and 1 to $7 \%$, respectively, and some remnant pancreatic le- sions developed in more than 10 years after initial resection of IPMN, indicating the importance of life-long surveillance.

Morphological classification and histopathologic grade of IPMN seem to be still important to predict patterns of recurrence or development of new lesions in the remnant pancreas after partial pancreatectomy for IPMN.

Tamura et al. [24] specifically analyzed the recurrence pattern after resection of MD-IPMN using pathological/ genomic analyses. Recurrence after partial pancreatectomy for MD-IPMN was observed in 6 of 49 patients (12\%), all of which were malignant IPMNs. Interestingly, examined 4 recurrent IPMNs had histological subtypes and KRAS/GNAS mutations identical to those of the initial lesions, despite the negative surgical margin at the initial operation. These findings suggested an unexpected recurrence pattern of MDIPMN as we called "monoclonal skip progression". Date et al. [36] confirmed the "monoclonal skip progression" of MD-IPMN using mRNA expression profiling.

Our previous studies [37-39], as well as others including a nationwide survey in Japan [40], which analyzed morphological/pathological subtypes of IPMN with concomitant PDAC, demonstrated the frequent occurrence of PDAC in the pancreas with BD-IPMN/gastric-type IPMN. Matsuda et al. [41] reported a significantly higher density of PanINs in the pancreas with IPMN and concomitant PDAC compared to PDAC without IPMN, which indicates the possible presence of undetectable precursor lesions in the remnant pan-

Table 3. Reports of Remnant Pancreatic Lesions after Resection of IPMN

\begin{tabular}{|c|c|c|c|c|c|c|c|c|}
\hline \multirow[b]{2}{*}{ Author } & \multirow[b]{2}{*}{ Journal } & \multirow[b]{2}{*}{ Year } & \multirow{2}{*}{$\begin{array}{l}\text { No. of } \\
\text { total } \\
\text { patient }\end{array}$} & \multirow{2}{*}{$\begin{array}{c}\text { No. of } \\
\text { patients with } \\
\text { noninvasive } \\
\text { IPMN }\end{array}$} & \multirow[b]{2}{*}{$\begin{array}{l}\text { Follow-up } \\
\text { period }\end{array}$} & \multicolumn{2}{|c|}{ Remnant pancreatic lesions } & \multirow{2}{*}{$\begin{array}{c}\text { Maximum } \\
\text { duration } \\
\text { between 1st } \\
\text { and } 2 \text { nd lesions }\end{array}$} \\
\hline & & & & & & $\begin{array}{c}\text { No. of total } \\
\text { patients }\end{array}$ & $\begin{array}{l}\text { No. of patients with } \\
\text { malignant lesions }\end{array}$ & \\
\hline $\begin{array}{l}\text { Schnelldorfer } \\
\text { et al. [28] }\end{array}$ & Arch Surg & 2008 & 208 & 145 & 3.2 years & 11 & 3 & 11 years \\
\hline Miller et al. [19] & НРB & 2011 & 243 & 243 & 73 months & $31\left(+38^{*}\right)$ & 4 (invasive IPMN) & 145 months \\
\hline Moriya et al. [30] & Arch Surg & 2012 & 203 & 160 & 40 months & $17\left(+14^{*}\right)$ & 4 & 127 months \\
\hline Frankel et al. [31] & НРВ & 2013 & 192 & 192 & 46 months & 40 & 3 & N/A \\
\hline Pea et al. [32] & Ann Surg & 2017 & 260 & 260 & $\mathrm{~N} / \mathrm{A}$ & 50 & 16 & 134 months \\
\hline Hirono et al. [33] & Surgery & 2016 & 257 & 172 & 53.5 months & 14 & 12 (IPMN 7/PDAC 5) & 71.6 months \\
\hline Miyasaka et al. [3] & Ann Surg & 2016 & 195 & 160 & 47 months & 29 & 13 (IPMN 6/PDAC 7) & 160 months \\
\hline Rezaee et al. [34] & НРB & 2016 & 616 & 433 & 28 months & 62 & 28 (IPMN 5/PDAC 23) & N/A \\
\hline Al Efishat et al. [35] & Ann Surg Oncol & 2018 & 319 & 299 & 42 months & 71 & 15 (IPMN 4/PDAC 11) & N/A \\
\hline
\end{tabular}

IPMN, intraductal papillary mucinous neoplasm; PDAC, pancreatic ductal adenocarcinoma; N/A, not assessed. *Residual lesions. 
creas after pancreatectomy for BD-IPMN.

Miyasaka et al. [3] comprehensively investigated the natural history of remnant pancreas after partial pancreatectomy for IPMN to identify factors associated with the metachronous development of high-grade dysplasia or invasive carcinoma of IPMN and concomitant PDAC, which we defined
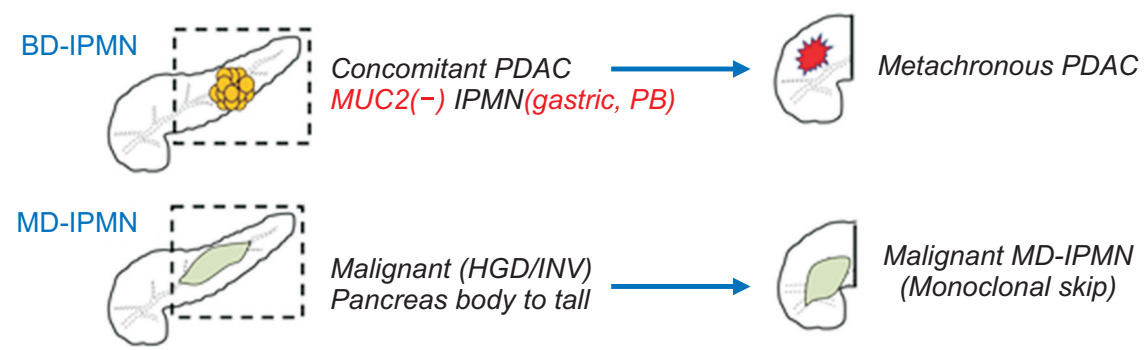

Malignant MD-IPMN (Monoclonal skip)

Others
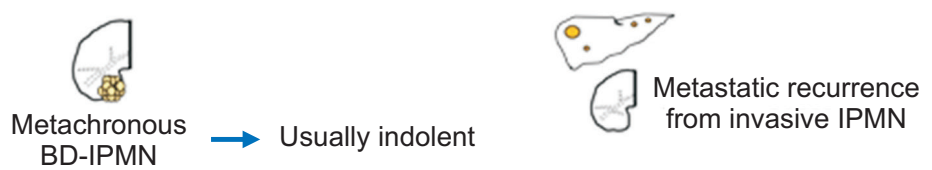
BD-IPMN
Resection of branch duct IPMNs

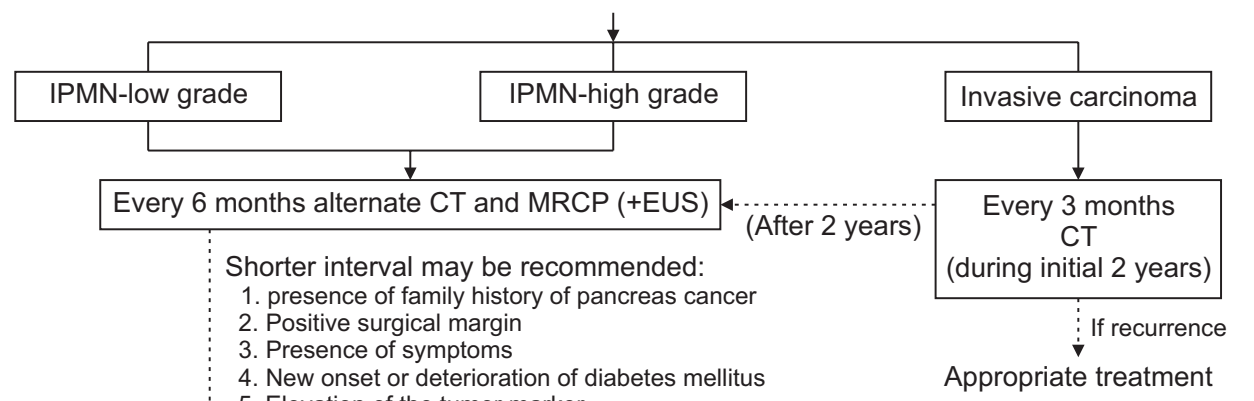

5. Elevation of the tumor marker

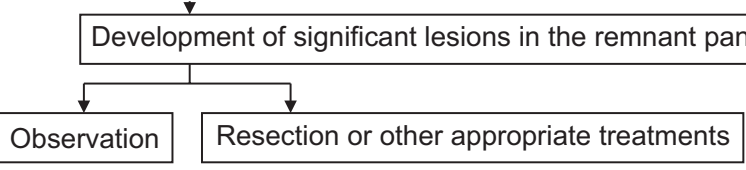

*Attention should also be paid to extra-pancreatic malignancies.

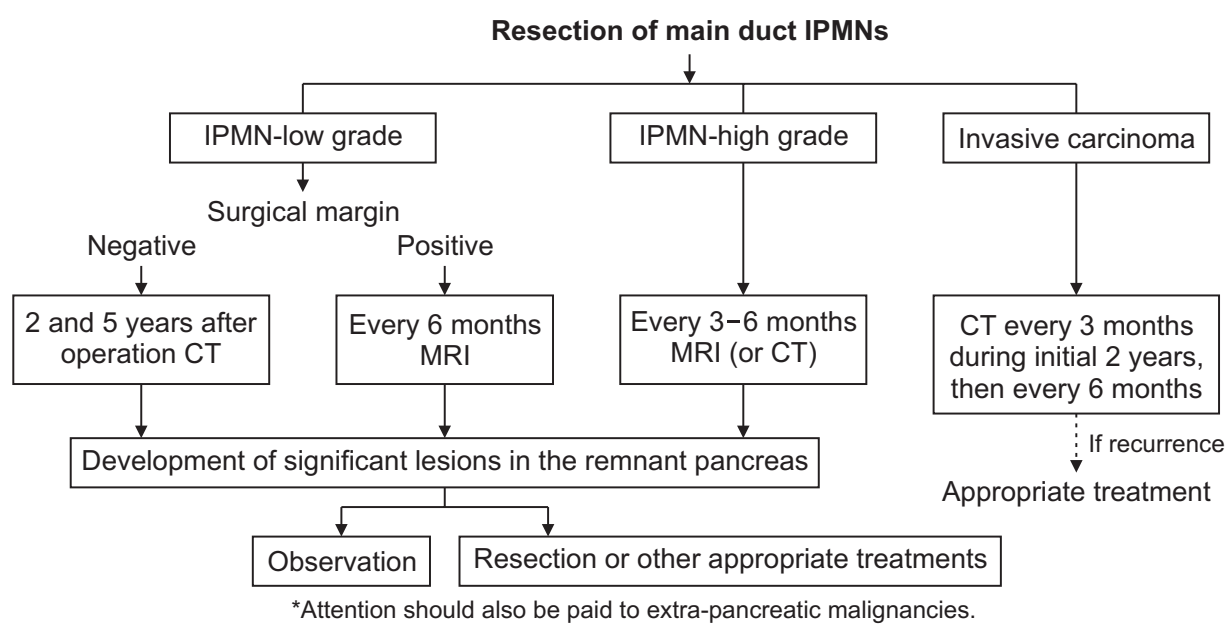

Fig. 3. Recurrence patterns after resection of IPMN based on characteristics of each morphological subtype of IPMN. Gastric type IPMN, gastric. Pancratobiliary type IPMN, PB. IPMN, intraductal papillary mucinous neoplasm; MD-IPMN, main duct IPMN; BD-IPMN, branch ductIPMN; PDAC, pancreatic ductal adenocarcinoma; HGD, high-grade dysplasia; INV, invasive carcinoma; $\mathrm{PB}$, pancreatobiliary type.

Fig. 4. A surveillance protocol after resection of BD-IPMN. IPMN, intraductal papillary mucinous neoplasm; BD-IPMN, branch duct-IPMN; MRCP, magnetic resonance cholangiography; CT, computed tomography; EUS, endoscopic ultrasound.

Fig. 5. A surveillance protocol after resection of MD-IPMN. IPMN, intraductal papillary mucinous neoplasm; MD-IPMN, main duct IPMN; CT, computed tomography; MRI, magnetic resonance imaging. 
as high-risk lesions. Multiple regression analyses identified the independent predictive factors including IPMNs located in the distal pancreas, HG/invasive IPMNs at index surgery, pancreatobiliary type IPMNs and presence of PDAC concomitant with IPMN as summarized in Fig. 3. The 5- and 10-year cumulative incidences of metachronous high-risk lesions in the remnant pancreas were $7.8 \%$ and $11.8 \%$, respectively.

\section{Surveillance Strategy after Pancreatectomy for BD/ MD-IPMN}

Based on characteristics of each morphological subtype relevant to postoperative surveillance described above, we proposed surveillance protocols after resection of IPMN (Figs. 4, 5) [42,43]. Surveillance interval was classified according to the histopathologic grade of IPMN resected at initial operation. The problem will be any protocol have the potential to fail to identify resectable high-risk lesions, and therefore, as proposed in Fig. 4, identification of high-risk individuals who need shorter interval surveillance is essential.

\section{CONCLUSIONS}

A proposed simple progression model of IPMN and slow growing biology appear to ensure the time window to resect IPMN before it becomes invasive carcinoma, resulting in a favorable prognosis in comparison to conventional PDAC. Because of the longer life expectancy after removal of IPMN, organ preserving surgery, without spoiling curability, need to be considered to preserve patients' quality of life. Remnant pancreas after partial pancreatectomy for IPMN seems to be at risk of development of "high-risk lesions", of which early detection and additional resection may benefit the patients. To diagnosis "high-risk lesions" at an earlier stage, physicians need to have insight into the unique biology of various types of IPMN.

\section{CONFLICTS OF INTEREST}

No potential conflict of interest relevant to this article was reported.

\section{AUTHOR'S CONTRIBUTIONS}

Conceptualization: Masafumi Nakamura. Supervision: Kohei Nakata, Masafumi Nakamura. Writing_original draft: Noboru Ideno. Writing-review \& editing: Kohei Nakata, Masafumi Nakamura.

\section{ORCID}

Noboru Ideno, https://orcid.org/0000-0003-4745-8417

Kohei Nakata, https://orcid.org/0000-0002-5717-8569

Masafumi Nakamura, https://orcid.org/0000-0002-6196-8643

\section{REFERENCES}

1. Tanaka M, Fernández-Del Castillo C, Kamisawa T, et al. Revisions of international consensus Fukuoka guidelines for the management of IPMN of the pancreas. Pancreatology 2017;17:738-753.

2. Basturk O, Hong SM, Wood LD, et al. A revised classification system and recommendations from the Baltimore Consensus Meeting for neoplastic precursor lesions in the pancreas. Am J Surg Pathol 2015;39:1730-1741.

3. Miyasaka Y, Ohtsuka T, Tamura K, et al. Predictive factors for the metachronous development of high-risk lesions in the remnant pancreas after partial pancreatectomy for intraductal papillary mucinous neoplasm. Ann Surg 2016;263:1180-1187.

4. Mino-Kenudson M, Fernández-del Castillo C, Baba Y, et al. Prognosis of invasive intraductal papillary mucinous neoplasm depends on histological and precursor epithelial subtypes. Gut 2011;60:1712-1720.

5. Yopp AC, Katabi N, Janakos M, et al. Invasive carcinoma arising in intraductal papillary mucinous neoplasms of the pancreas: a matched control study with conventional pancreatic ductal adenocarcinoma. Ann Surg 2011;253:968- 
974.

6. Hirono S, Shimizu Y, Ohtsuka T, et al. Recurrence patterns after surgical resection of intraductal papillary mucinous neoplasm (IPMN) of the pancreas; a multicenter, retrospective study of 1074 IPMN patients by the Japan Pancreas Society. J Gastroenterol 2020;55:86-99.

7. Sohn TA, Yeo CJ, Cameron JL, et al. Intraductal papillary mucinous neoplasms of the pancreas: an updated experience. Ann Surg 2004;239:788-797; discussion 797-799.

8. Furukawa T, Hatori T, Fujita I, et al. Prognostic relevance of morphological types of intraductal papillary mucinous neoplasms of the pancreas. Gut 2011;60:509-516.

9. European Study Group on Cystic Tumours of the Pancreas. European evidence-based guidelines on pancreatic cystic neoplasms. Gut 2018;67:789-804.

10. Vege SS, Ziring B, Jain R, Moayyedi P; Clinical Guidelines Committee; American Gastroenterology Association. American gastroenterological association institute guideline on the diagnosis and management of asymptomatic neoplastic pancreatic cysts. Gastroenterology 2015;148:819-822; quize812-813.

11. Oyama H, Tada M, Takagi K, et al. Long-term risk of malignancy in branch-duct intraductal papillary mucinous neoplasms. Gastroenterology 2020;158:226-237.e225.

12. Schmidt CM, White PB, Waters JA, et al. Intraductal papillary mucinous neoplasms: predictors of malignant and invasive pathology. Ann Surg 2007;246:644-651; discussion 651-654.

13. Rodriguez JR, Salvia R, Crippa S, et al. Branch-duct intraductal papillary mucinous neoplasms: observations in 145 patients who underwent resection. Gastroenterology 2007;133:72-79; quiz 309-310.

14. Waters JA, Schmidt CM, Pinchot JW, et al. CT vs MRCP: optimal classification of IPMN type and extent. J Gastrointest Surg 2008;12:101-109.

15. Mori Y, Ohtsuka T, Kono H, et al. Management strategy for multifocal branch duct intraductal papillary mucinous neoplasms of the pancreas. Pancreas 2012;41:1008-1012.

16. Matthaei H, Norris AL, Tsiatis AC, et al. Clinicopathological characteristics and molecular analyses of multifocal intraductal papillary mucinous neoplasms of the pancreas. Ann Surg 2012;255:326-333.

17. Shi C, Klein AP, Goggins M, et al. Increased prevalence of precursor lesions in familial pancreatic cancer patients. Clin Cancer Res 2009;15:7737-7743.

18. Bartsch DK, Dietzel K, Bargello M, et al. Multiple small "imaging" branch-duct type intraductal papillary mucinous neoplasms (IPMNs) in familial pancreatic cancer: indicator for concomitant high grade pancreatic intraepithelial neoplasia? Fam Cancer 2013;12:89-96.

19. Skaro M, Nanda N, Gauthier C, et al. Prevalence of germline mutations associated with cancer risk in patients with intraductal papillary mucinous neoplasms. Gastroenterology 2019;156:1905-1913.

20. He J, Cameron JL, Ahuja N, et al. Is it necessary to follow patients after resection of a benign pancreatic intraductal papillary mucinous neoplasm? J Am Coll Surg 2013;216:657-665; discussion 665-667.

21. Kang CM, Choi SH, Kim SC, Lee WJ, Choi DW, Kim SW. Predicting recurrence of pancreatic solid pseudopapillary tumors after surgical resection: a multicenter analysis in Korea. Ann Surg 2014;260:348-355.

22. Dhar VK, Merchant NB, Patel SH, et al. Does surgical margin impact recurrence in noninvasive intraductal papillary mucinous neoplasms?: a multi-institutional study. Ann Surg 2018;268:469-478.

23. Marchegiani G, Mino-Kenudson M, Sahora K, et al. IPMN involving the main pancreatic duct: biology, epidemiology, and long-term outcomes following resection. Ann Surg 2015;261:976-983.

24. Tamura K, Ohtsuka T, Ideno N, et al. Treatment strategy for main duct intraductal papillary mucinous neoplasms of the pancreas based on the assessment of recurrence in the remnant pancreas after resection: a retrospective review. Ann Surg 2014;259:360-368.

25. Hara T, Yamaguchi T, Ishihara $T$, et al. Diagnosis and patient management of intraductal papillary-mucinous tumor of the pancreas by using peroral pancreatoscopy and intraductal ultrasonography. Gastroenterology 2002;122:34-43.

26. Nagayoshi Y, Aso T, Ohtsuka T, et al. Peroral pancreatoscopy using the SpyGlass system for the assessment of 
intraductal papillary mucinous neoplasm of the pancreas. J Hepatobiliary Pancreat Sci 2014;21:410-417.

27. Ohtsuka T, Gotoh Y, Nakashima Y, et al. Role of SpyGlass-DSTM in the preoperative assessment of pancreatic intraductal papillary mucinous neoplasm involving the main pancreatic duct. Pancreatology 2018;18:566-571.

28. Schnelldorfer T, Sarr MG, Nagorney DM, et al. Experience with 208 resections for intraductal papillary mucinous neoplasm of the pancreas. Arch Surg 2008;143:639646; discussion 646.

29. Miller JR, Meyer JE, Waters JA, et al. Outcome of the pancreatic remnant following segmental pancreatectomy for non-invasive intraductal papillary mucinous neoplasm. HPB (Oxford) 2011;13:759-766.

30. Moriya T, Traverso W. Fate of the pancreatic remnant after resection for an intraductal papillary mucinous neoplasm: a longitudinal level II cohort study. Arch Surg 2012;147:528-534.

31. Frankel TL, LaFemina J, Bamboat ZM, et al. Dysplasia at the surgical margin is associated with recurrence after resection of non-invasive intraductal papillary mucinous neoplasms. HPB (Oxford) 2013;15:814-821.

32. Pea A, Yu J, Rezaee N, et al. Targeted DNA sequencing reveals patterns of local progression in the pancreatic remnant following resection of intraductal papillary mucinous neoplasm (IPMN) of the pancreas. Ann Surg 2017;266:133-141.

33. Hirono S, Kawai M, Okada K, et al. Long-term surveillance is necessary after operative resection for intraductal papillary mucinous neoplasm of the pancreas. Surgery 2016;160:306-317.

34. Rezaee N, Barbon C, Zaki A, et al. Intraductal papillary mucinous neoplasm (IPMN) with high-grade dysplasia is a risk factor for the subsequent development of pancreatic ductal adenocarcinoma. HPB (Oxford) 2016;18:236-246.

35. Al Efishat M, Attiyeh MA, Eaton AA, et al. Progression patterns in the remnant pancreas after resection of noninvasive or micro-invasive intraductal papillary mucinous neoplasms (IPMN). Ann Surg Oncol 2018;25:1752-1759.

36. Date K, Ohtsuka T, Fujimoto T, et al. Molecular evidence for monoclonal skip progression in main duct intraductal papillary mucinous neoplasms of the pancreas. Ann Surg 2017;265:969-977.

37. Ingkakul T, Sadakari Y, Ienaga J, Satoh N, Takahata S, Tanaka M. Predictors of the presence of concomitant invasive ductal carcinoma in intraductal papillary mucinous neoplasm of the pancreas. Ann Surg 2010;251:70-75.

38. Yamaguchi K, Kanemitsu S, Hatori T, et al. Pancreatic ductal adenocarcinoma derived from IPMN and pancreatic ductal adenocarcinoma concomitant with IPMN. Pancreas 2011;40:571-580.

39. Ideno $\mathrm{N}$, Ohtsuka $\mathrm{T}$, Kono $\mathrm{H}$, et al. Intraductal papillary mucinous neoplasms of the pancreas with distinct pancreatic ductal adenocarcinomas are frequently of gastric subtype. Ann Surg 2013;258:141-151.

40. Kobayashi G, Fujita N, Maguchi H, et al. Natural history of branch duct intraductal papillary mucinous neoplasm with mural nodules: a Japan Pancreas Society multicenter study. Pancreas 2014;43:532-538.

41. Matsuda R, Miyasaka Y, Ohishi Y, et al. Concomitant intraductal papillary mucinous neoplasm in pancreatic ductal adenocarcinoma is an independent predictive factor for the occurrence of new cancer in the remnant pancreas. Ann Surg 2020;271:941-948.

42. Ohtsuka T, Tanaka M. Postoperative surveillance of main duct IPMN. In: Tanaka M, ed. Intraductal papillary mucinous neoplasm of the pancreas. Tokyo: Springer, 2014:181-188.

43. Ohtsuka T, Tanaka M. Postoperative surveillance of branch duct IPMN. In: Tanaka M, ed. Intraductal papillary mucinous neoplasm of the pancreas. Tokyo: Springer, 2014:189-199. 